Pain Physician. 2004;7:295-299, ISSN 1533-3159

\title{
EVolving Concepts in the Understanding of Cervical facet Joint Pain
}

\begin{abstract}
Rinoo V. Shah, MD, and Alan D. Kaye, MD
Surgeons loathe the concept of masking a painful, acute abdomen with analgesics: serial abdominal exams are keys to surgical diagnosis and planning (1). Pain physicians have a softer spot. Sedatives and analgesics are given to patients undergoing interventional pain

Although the central objective of Manchikanti et al's paper was to evaluate how intravenous fentanyl, midazolam, or even, placebo can confound the interpretation of a 'diagnostic' medial branch block, this paper creates a curious byproduct and uncovers a shocking problem.
\end{abstract} procedures, because these patients are in chronic pain and demand no less than to be free of chronic pain (2). However, could intravenous sedation, anxiolysis, and analgesia confound the interpretation of a 'diagnostic' block?

Manchikanti and colleagues (3) present an important paper that formally evaluates this question, with respect to cervical medial branch blocks. If pain relief of $>50 \%$ is used as a criterion, then intravenous fentanyl, midazolam, and saline (placebo) could lead to false positives. Use of stricter pain relief criteria, $>80 \%$ reduction in pain and the ability to perform previously painful neck movements with ease, can overcome these confounding factors. We applaud Manchikanti et al's recommendations that intravenous sedation need not be abandoned, but should be used judiciously. In the absence of sedation, patients may move or become combative. These are the realities of clinical practice, but unfortunately procedure validity may be compromised. The prudent use of short acting sedatives and analgesics, along with careful monitoring, seems appropriate.

From Department of Anesthesiology and Pain Services, Texas Tech University Health Sciences Center, Lubbock, Texas. Dr. Shah is an Assistant Professor in the Department Anesthesiology and Pain Services. Dr. Kaye is the Chairman and Professor of the Department of Anesthesiology and Pain Services and Professor in the Department of Pharmacology. Address Correspondence: Rinoo V. Shah, MD, 4430 South Loop 289, Lubbock, TX 79414

E-mail: rinoo_shah@yahoo.com

Funding: There was no external funding in preparation of this manuscript.

Conflict of Interest: None

\section{A Curious Byproduct}

One curious byproduct of this study is that $40 \%$ of the patients, in the saline control group, achieved a 'relaxed' state. Pre-procedure opioid or benzodiazepine use must have been homogeneous among all three groups, since the study's inclusion criteria and randomization were not based on these factors. Nonetheless, patients in Group II and Group III required an average of $3.5 \pm 1.2$ milligrams of midazolam and $160 \pm 50$ micrograms of fentanyl to achieve a 'relaxed' state, without any complications. This implies that patients in all three groups were opioid or benzodiazepine tolerant. So, how could placebo have such an impact on $40 \%$ of the patients in the intravenous saline group (Group I)? Is placebo really that powerful? Could placebo be incorporated into clinical practice, as a 'sedative and analgesic', to improve patient safety and turnover or is placebo ethically undesirable?

Functional magnetic resonance imaging studies demonstrate that placebo alters the experience of pain and expectations about pain relief (4). Analgesia induced by placebo is related to decreased activity in pain-sensitive brain regions, such as the thalamus, insula, and anterior cingulate cortex (ACC) (4). Activity increases in the prefrontal cortex when pain is anticipated (4). The rostral anterior cingulate cortex (rACC) and anterior insula are implicated in the affective, but not the sensory aspects of pain (5). Activity in the posterior insula/secondary somatosen- sory cortex, the sensorimotor cortex (SI/ $\mathrm{MI}$ ), and the caudal ACC receive the sensory aspects of pain (5). Placebo and opioid analgesia share similar neural mechanisms, specifically at the rACC and brainstem based on PET scanning (6).

Despite the neurobiological complexity, placebo analgesia is similar to opioid analgesia. Placebo can be easily induced, simply by expecting that a procedure will alleviate pain. Perhaps, placebo is not ethically undesirable and could be used as a tool in the procedure suite.

\section{A Shocking Problem Unearthed}

What if there is no such thing as a 'diagnostic' medial branch block or for that matter, a 'diagnostic' nerve block?

The medial branches of the segmental, cervical spinal nerves have gained popularity over the past twenty years. Much has been written about their intimate anatomic relationship to the cervical zygapophyseal joints $(7,8)$, their physiological relevance as a neural relay in patients with chronic neck pain $(7,9,10)$, and their role in estimating the prevalence of chronic pain mediated by the cervical z-joints $(7,11)$.

As our understanding of cervical zjoint pain has increased, the relevance of patient history, physical exam, and imaging studies has diminished $(7,12,13)$. Evidence suggests that chronic zygapophyseal joint pain need only be diagnosed by cervical medial branch blocks $(7,12-17)$, but false positives should be reduced. Uncontrolled blocks lead to a high false positive rate, so placebo controlled blocks are advised (7, 11-17). However, as discussed earlier, placebo is still considered to be unethical $(15,16)$. Comparative local anesthetic blocks have been advocated instead, after studies have proven them to be effective surrogates for placebo controlled blocks $(15,16)$. 
'If pain is relieved [by blocking the medial branch or the z-joint itself], the joint may be considered prima facie to be the source of pain' (12). This seemingly logical statement must be completely true in order for the moniker 'diagnostic' to survive as an adjective to medial branch block or, for that matter, nerve block. Advances in our scientific understanding of pain and clinical paradoxes challenge the veracity of the term diagnostic medial branch block.

1. Does the average analgesic response of a placebo group reliably predict the analgesic response in a specific individual? Is the placebo response in an individual completely reproducible if the placebo intervention is repeated?

This question is important when we wish to counsel patients about the results of their 'diagnostic' medial branch block. The Taxonomy Committee of the International Association for the Study of Pain defined pain as "an unpleasant sensory and emotional experience associated with actual or potential tissue damage" (18). The corollary is that pain relief would have to be defined in these terms. Simply, pain and pain relief are experiences that are unique to each individual and cannot always be predicted.

Interventional pain studies typically use demographic factors, such as age, gender, chronicity of pain, and prior interventions, to establish that the placebo and intervention group are similar. These data are snapshots of the patient's present and recent past: they do not represent all the factors that influence an individual's pain perception. Furthermore, demographic data are not mentioned in the IASP definition of pain.

Ideally, the placebo and intervention group should be similar in terms of all factors that may impact pain, such as familial upbringing, schooling, religion, psychological experiences, injuries, genetic make-up, and cultural heritage. Of course, the use of such factors to ensure homogeneity between both groups would be implausible. To do anything less, however, calls into question the relevance or the need for placebo-controlled studies in interventional pain.

The answer to the second question can be found in Manchikanti et al's paper. All study patients were diagnosed, in the past, to have cervical facet joint pain. This was confirmed with comparative local anesthetic, medial branch blocks. Compar- ative local anesthetic blocks, supposedly, have good construct validity and can reduce the rate of false positives (14-16). In the current study, the subjects did not receive medial branch blocks. They only received intravenous agents for the study. However, all the patients had neck pain of at least 1 year duration, with confirmed evidence of facet joint pain by controlled comparative local anesthetic blocks and were responding to therapeutic medial branch blocks. They were presenting for repeat treatment after a significant period of symptom relief. Thus, there was proven evidence of facet joint pain. Consequently, relief of this pain was tested with multiple agents.

Price et al (19) studied diagnostic, sympathetic blocks in patients with complex regional pain syndrome. This paper raised several concerns about block interpretation with respect to placebo. The placebo response can be influenced by preprocedure anxiety, the desire for pain relief, expectations about the block, and the perceived invasiveness and elaborateness of the procedure (19). Pre-procedure anxiety could enhance sympathetic efferent outflow, which can augment pre-procedure pain (19). These factors were not addressed when the construct validity of comparative local anesthetic blocks was investigated (16).

Overall, the complexity of the placebo response creates doubts about the term 'diagnostic' medial branch block.

2. If a 'diagnostic' medial branch block successfully reduces pain and efforts are made to reduce false positives, can we accept at face validity that the facet joint is the source of (nociceptive) pain or could peripheral and central (neuropathic) pain mechanisms be involved?

Zygapophyseal joints are synovially-lined, diarthroidal, load bearing joints with hyaline cartilage (20). They can be subject to trauma and degenerative arthropathy.

Let us start with a simple analogy and consider another synovially-lined, diarthroidal joint that can be injured: the knee. Is it possible that an orthopedic surgeon would operate on a patient that has chronic knee pain and not perform a clinical evaluation nor study the patient's imaging. This is doubtful (21-24). Is it possible that an orthopedic surgeon would rely on a combined sciatic and femoral nerve block, which can anesthetize the entire knee joint (25), to decide whether or not to operate? This is doubtful.

Biomechanical studies demonstrate that injury to cervical z-joints leads not only to nociceptive pain, but can lead to neuropathic pain, by injuring the joint and proximate neural structures (26). It is conceivable that the medial branches could be injured because of cervical trauma or degenerative processes. In this case, pain relief following a medial branch block would be prima facie evidence that the traumatized nerve, itself, is the pain generator and not the joint.

Neuropathic pain is both spontaneous (stimulus independent) and evoked (stimulus dependent), which lends credence to the concept that the history and physical are irrelevant, i.e., provocative activities are not needed to diagnose $z$-joint pain. However, if the pain emanated only from the z-joint (nociceptive), one would expect the history and physical to be relevant: increased pain with provocative or load bearing maneuvers compared to pain at rest. In fact, biomechanical studies suggest that neck extension should provoke neck pain following whiplash (27).

Since there is no evidence that cervical zygapophyseal joint pain can be diagnosed by clinical examination or by medical imaging (17), cervical z-joint pain is arguably neuropathic rather than nociceptive.

\section{Peripheral Sensitization}

Injury to peripheral tissues can lead to sensory disturbances characterized by prolonged pain, increased sensitivity to painful stimuli (hyperalgesia), and pain following innocuous stimuli (allodynia). The peripheral afferent neuronal discharge from tissue injury can lead to central nervous system hyperexcitability and lead to worse pain following tissue injury (28).

Gordon et al (28) in a parallel group, double blind, placebo controlled study compared the ability of injections of intra-oral saline versus a long acting local anesthetic, (bupivacaine $0.5 \%$ ) to reduce post-operative pain. Selected patients underwent oral surgery with general anesthesia. After awakening and after the anesthetic had worn off, the patients were enrolled in this study. Curiously, there was no difference in pain relief between the placebo and bupivacaine groups at 24 hours. There was a significant reduc- 
tion in pain at 48 hours, long after the local anesthetic had worn off. Hence, even if injections are administered subsequent to tissue injury (not preemptive analgesia), pain relief can be significant, but delayed (28). The authors conclude that blockade of this peripheral nociceptive input after tissue injury can reduce post-injury pain long after the local anesthetic has worn off. This suggests that peripheral blocks reduce central hyperexcitability and consequently, pain (28). This study contradicts the premise that a long acting local anesthetic block of the medial branch should provide immediate relief that can be sustained for 24 hours (15-16).

\section{Central Sensitization}

Central sensitization does exist in patients with chronic pain following whiplash injury and may be maintained by peripheral stimulation $(29,30)$. This central hypersensitization was not maintained by peripheral input from painful and tender muscles (30). The central sensitization can be maintained by low intensity nociceptive and innocuous peripheral stimulation (29). Chronic neck pain, following whiplash without detectable tissue damage, may be due to spinal hypersensitivity. This further supports the hypothesis that central sensitization may be potentiated by input from the cervical medial branches. Hyperalgesia occurs if these medial branches are mildly provoked and allodynia occurs when they process usually painless stimuli.

Synovially lined joints have Ruffini nerve endings with central projections to the thalamus and sensorimotor cortex, suggesting that these joints are responsible for proprioception, i.e., joint position sense (31). These central projections occur via small myelinated fibers (31). Paqueron et al (31) noted that the abolition of proprioception corresponded to the onset of phantom limb sensations, during peripheral nerve blocks; phantom-limb sensations coincided with block of $\mathrm{C}$ and A-delta fibers.

This suggests that $\mathrm{C}$ and A-delta fibers are not only responsible for transmitting pain, but proprioception, limb size, and limb shape perception as well (32). Perhaps this is why joint position sense could be perceived as painful, following neck injury.

Swelling illusion has recently been demonstrated to be an early indicator of the success of a peripheral nerve block
(32). Since swelling illusion depends on impairment of small sensory fibers (32) as does pain relief, perhaps this methodology could be used to validate comparative local anesthetic blocks: do medial branch blocks lead to perceptual alterations in the size of the neck, in addition to relieving pain? This type of external validation would be useful, since there are no other physiological means to test for block adequacy, including superficial examination (33).

\section{Central and Peripheral Processes}

Manchikanti et al's paper lends support to the hypothesis that both central and peripheral sensitization occurs in cervical facet pain.

Central sensitization may occur by inducing Long Term Potentiation (LTP) at the synapses between C-fiber afferents and superficial spinal dorsal horn neurons (34). In rats, low dose fentanyl infusions (12-48 mcg/kg/hour) block LTP, but not higher anti-nociceptive fentanyl doses (96-12 mcg/k/hour) (34). Manchikanti et al's fentanyl group required $110-210 \mathrm{mcg}$. Let us assume that the average patient is $70 \mathrm{~kg}$ and that procedure completion times range between 10 to 60 minutes. Then, the fentanyl delivery rate would vary between $1.57 \mathrm{mcg} / \mathrm{kg} / \mathrm{hr}(110 \mathrm{mcg} /$ 60 minutes/ $70 \mathrm{~kg}$ x 60 minutes/1 hour) to $18 \mathrm{mcg} / \mathrm{kg} / \mathrm{hour}(210 \mathrm{mcg} / 10$ minutes/ $70 \mathrm{~kg}$ x 60 minutes/1 hour). These values are low dose and could block central sensitization. In fact, the fentanyl group had the greatest proportion of pain relief, when compared to placebo. A clinical study confirmed the effects of fentanyl on central sensitization. Epidural fentanyl increased temporal summation threshold and could conceivably prevent and treat central hypersensitivity (35). The peripheral action of opioids may also account for the increased analgesic effect in the fentanyl group. $\mathrm{Mu}$ opioid agonists have been demonstrated to have a peripheral effect (36).

Collectively, these neurobiological pain processes question the ideology that pain relief following a cervical medial branch block is proof that the z-joint is the de facto pain generator.

3. Can placebo-controlled medial branch blocks or their surrogate, comparative local anesthetic blocks, be confounded by pharmacological or technical factors?

In their study validating compara- tive local anesthetic blocks of the cervical medial branches, Barnsley et al (16) used the premise that bupivacaine is longer acting than lignocaine (lidocaine). During peripheral nerve blocks, bupivacaine and ropivacaine have significantly longer durations of action, when compared to lidocaine and mepivacaine $(37,38)$. In fact, ropivacaine may provide post-operative analgesia for 12-14 hours after a combined sciatic-femoral nerve block, whereas mepivacaine may provide blockade for 5-7 hours (38). However, simply correlating local anesthetic duration of action with duration of pain relief seems overly simplistic. In fact, in Barnsley et al's study (16), almost $40 \%$ of patients had responses to cervical medial branch blocks that did not meet the expected duration of either one or both local anesthetics (lidocaine, bupivacaine).

Price et al (19) compared the painrelieving effects of local anesthetics (lidocaine/bupivacaine) against saline during sympathetic ganglion blocking procedures. Both reduced peak pain intensities by $70 \%$ at 30 minutes post-procedure: no statistically significant difference was noted. In striking contrast, the mean duration of pain relief was longer with local anesthetic (90 hours), when compared to saline (20 hours). This duration of action is unusually long for local anesthetics.

If pain persists after a neural pathway is injected with local anesthetic, then that pathway may still be involved, because neural blockade is not an all-or-none response (33). Hogan and Abram (33) provide several examples. Somatosensory potentials can still be evoked in an anesthetized region, even if there is complete insensitivity to touch and pain $(33,39)$. Sympathetic efferent output might not be completely blocked in an area of complete somatic blockade $(33,40)$.

Local anesthetics have a differential effect, depending on neural fiber type, fiber size, and drug diffusion (33). Small size C fibers, that transmit pain are typically blocked first by lidocaine and bupivacaine (41) and their blockade lasts longer than those of other sensory, (A-beta) and motor, (A-alpha) fibers (41). Other studies have demonstrated that the intrinsic sensitivity of fiber types to local anesthetics is $A>B>C$ (33). Different nerve groups have a significant overlap in the necessary concentration for nerve blockade and this may impact the ability to dis- 
cern a differential nerve block based on fiber size (42). This is relevant to cervical medial branch blocks because several fiber types may be responsible for transmitting pain and these fibers may not necessarily be blocked as expected.

The duration of action depends on some physical properties of the local anesthetic, such as protein binding and metabolism (44). These factors probably remain the same if only a short period of time elapses between blocks. Similarly, systemic absorption following low volume blocks is probably negligible: there are probably no analgesic effects from systemic absorption of local anesthetic following cervical medial branch blocks.

Three consecutive nodes of Ranvier must be blocked to prevent conduction (43). The small volumes used for comparative local anesthetic blocks were chosen to ensure no spread to the exiting spinal nerve (16), but can those authors be certain that 3 nodes of Ranvier were being consistently blocked? Nerve length influences anesthetic potency (33). Injection rate and the microanatomic properties of nerve fibers may affect intraneural spread of local anesthetic $(45,46)$. Inadvertent intraneural injections can cause significant centripetal spread (45), which could theoretically lead to unrecognized segmental spinal nerve blockade. Lidocaine has to be within $1 \mathrm{~mm}$ and $0.5 \mathrm{~mm}$ of a rabbit sciatic nerve to effectively block $\mathrm{C}$ and A delta fibers, respectively (46). When injections are performed in such close proximity, even $0.1 \mathrm{ml}$ can spread more than 2 centimeters (47). This type of spread, during a cervical medial branch block, would imply that the spinal nerve could be blocked.

The appropriate concentration and dose required to block cervical medial branches are not known from a physiological standpoint. In the rabbit sciatic nerve, the concentration is more important than volume or dose for successful block. Smaller volumes with higher local anesthetic concentration produce blocks of greater depth and duration compared to larger volume and lower concentrations, with the same dose (46). Hence, the precise amount and concentration of local anesthetic for cervical medial branch blocks has not been validated.

Vascular uptake may shorten block duration. The intraneural concentration of a local anesthetic following a peripheral nerve block is relevant: this value corre- sponds to the degree of functional blockade (48). Nonetheless, only a small fraction of the applied local anesthetic becomes intraneural (46). If small volumes or doses of local anesthetic are used, vascular uptake impacts the amount of drug reaching the intraneural compartment (46). Furthermore, local anesthetics exert complex effects on vascular tone and hence, the rate of vascular uptake.

If high concentrations of local anesthetic are used or high doses of local anesthetic become intraneural, irreversible conduction block may occur (49). This may partially account for why some patients in Barnsley et al's study (16) had unusually prolonged responses to medial branch blockade.

Even if all these factors are taken into account, several perplexing problems with local anesthetic blocks persist. Dorsal root ganglia abnormally and spontaneously fire off impulses in response to nerve injury $(33,50)$. An injured medial branch could induce spontaneous DRG activity. Blocking the cervical medial branch in this setting, would not provide pain relief. Should we consider this to be a true negative? Another questionable assumption is that only afferent nociception needs to be blocked. When a nerve is injured, however, orthodromic and antidromic bursts are generated. In the case of a sensory nerve, antidromic impulses travel toward the tissue injury. Injured sensory nerves may peripherally release a soup of painful, chemical mediators. In this case, pain perception may be altered by a block distal to the injury. Peripheral blockade of the sciatic nerve has provided relief to patients with documented lumbosacral radiculopathy $(33,51)$. Perhaps the most perplexing phenomenon with peripheral local anesthetic blocks is the ability to block a "distal" nonexistent joint (52). Imagine this statement, following a successful sciatic nerve block: "the abolition of phantom ankle pain in a patient with an above the knee amputation is 'prima facie' evidence that the nonexistent ankle is the pain generator" $\mathrm{Al}$ though there is solid evidence that neck pain can be relieved by blockade of medial branch nerves, which innervate the facet joints, the precise mechanisms of pain relief remain unclear. Indeed, "facet" pain may arise from structures other than the facet joints per se. Alternate sources of pain include soft tissue structures, such as muscle and fascia, and the nerves themselves. Indeed, the well-known phenom- enon of pain relief outlasting the expected duration of local anesthetic effect supports the argument that facet pain has a neuropathic component.

In summary, Manchikanti et al's paper is outstanding and resolves some controversies (the use of sedation), but raises others (is the adjective "diagnostic" appropriate for medial branch or other nerve blocks). As interventional pain physicians, we must pay heed that our procedures do not simply fit into an anatomic construct, but into a vastly more complex neurobiological construct. Overall, facet joint pain exists, but diagnosing facet joint pain with medial branch blocks faces many challenges.

\section{Author Affiliation: \\ Rinoo V. Shah, MD \\ Assistant Professor \\ Department of Anesthesiology and \\ Pain Services \\ International Pain Institute \\ Texas Tech University Health Sciences \\ Center \\ 4430 South Loop 289 \\ Lubbock, Texas 79414 \\ E-mail: rinoo_shah@yahoo.com \\ Alan D. Kaye, MD \\ Chiarman and Professor \\ Department of Anesthesiology and \\ Pain Services \\ Professor of Pharmacology \\ Texas Tech University Health \\ Sciences Center \\ 4430 South Loop 289 \\ Lubbock, Texas 79414}

\section{RefERENCES}

1. Nissman SA, Kaplan LJ, Mann BD. Critically reappraising the literature-driven practice of analgesia administration for acute abdominal pain in the emergency room prior to surgical evaluation. Am J Surg 2003;18: 291-296.

2. Manchikanti L, Manchikanti KN, Damron KS et al. Effectiveness of cervical medical branch blocks in chronic neck pain: A prospective outcome study. Pain Physician 2004; 7:195-202.

3. Manchikanti L, Pampati V, Damron K et al. A randomized, prospective, double blind, placebo-controlled evaluation of the effect of sedation on diagnostic validity of cervical facet joint pain. Pain Physician 2004; 7:301-309.

4. Wager TD, Rilling JK, Smith EE et al. Placebo-induced changes in FMRI in the anticipation and experience of pain. Science 2004; 303:1162-1167.

5. Singer T, Seymour B, O'Doherty J et al. 
Empathy for pain involves the affective but not sensory components of pain. Science 2004; 303:1157-1162.

6. Petrovic P, Kalso E, Petersson KM et al. Placebo and opioid analgesia-- imaging a shared neuronal network. Science 2002; 295:1737-1740.

7. Boswell MV, Singh V, Staats P et al. Accuracy of precision diagnostic blocks in the diagnosis of chronic spinal pain of facet or zygapophysial joint origin: A systematic review. Pain Physician 2003; 6: 449-456.

8. Bogduk N. The clinical anatomy of the cervical dorsal rami. Spine 1982; 7:319-330.

9. Bogduk N. Neck pain. Aust Fam Physician 1984; 13:26-30.

10. Bogduk N, Marsland A. The cervical zygapophysial joints as a source of neck pain. Spine 1988; 13:610-617.

11. Lord SM, Barnsley L, Wallis BJ. Chronic cervical zygapophysial joint pain after whiplash. A placebo-controlled prevalence study. Spine 1996; 21:1737-1744.

12. Bogduk N. International Spinal Injection Society Guidelines for the Performance of Spinal Injection Procedures: Part 1: Zygapophysial Joint Blocks. Clin J Pain 1997; 13:292-297.

13. Manchikanti L, Boswell MV, Singh V et al. Prevalence of facet joint pain in chronic spinal pain of cervical, thoracic, and lumbar regions. BMC Musculoskelet Disord 2004; 5:15.

14. Barnsley L, Lord S, Wallis B et al. Falsepositive rates of cervical zygapophysial joint blocks. Clin J Pain 1993; 9:124-130.

15. Lord SM, Barnsley L, Bogduk N. The utility of comparative local anesthetic blocks versus placebo-controlled blocks for the diagnosis of cervical zygapophysial joint pain. Clin J Pain 1995; 11:208-213.

16. Barnsley L, Lord S, Bogduk N. Comparative local anesthetic blocks in the diagnosis of cervical zygapophysial joints pain. Pain 1993; 55:99-106

17. Manchikanti L, Singh V, Rivera JJ et al. Prevalence of cervical facet joint pain in chronic neck pain. Pain Physician 2002; 5:243-249.

18. Mersky H. Classification of chronic pain, descriptions of chronic pain syndromes and definitions of pain terms. Pain 1986; 3:S10-S24.

19. Price DD, Long S, Wilsey B et al. Analysis of peak magnitude and duration of analgesia produced by local anesthetics injected into sympathetic ganglia of complex regional pain syndrome patients. Clin J Pain 1998; 14:216-226.

20. Shah RV, Lutz GE. Lumbar intraspinal synovial cysts: conservative management and review of the world's literature. Spine J 2003; 3:479-488.

21. Calmbach WL, Hutchens M. Evaluation of patients presenting with knee pain: Part I. History, physical examination, radiographs, and laboratory tests. Am Fam Physician 2003; 68:907-912.
22. Jackson JL, O’Malley PG, Kroenke K. Evaluation of acute knee pain in primary care. Ann Intern Med 2003; 139:575-588.

23. Hammer $A$. $A B C$ of rheumatology: Pain in the hip and knee. BMJ 2004; 328:10671069.

24. Felson DT. - An update on the pathogenesis and epidemiology of osteoarthritis. Radiol Clin North Am 2004; 42:1-9.

25. Williams BA, Kentor ML, Vogt MT et al. Femoral-sciatic nerve blocks for complex outpatient knee surgery are associated with less postoperative pain before sameday discharge: a review of 1,200 consecutive cases from the period 1996-1999. Anesthesiology 2003; 98:1206-1213.

26. Cusick JF, Pintar FA, Yoganandan N. Whiplash syndrome: kinematic factors influencing pain patterns. Spine 2001; 26:12521258.

27. Yoganandan N, Pintar FA, Cusick JF. Biomechanical analyses of whiplash injuries using an experimental model. Accid Anal Prev 2002; 34:663-671.

28. Gordon SM, Dionne RA, Brahim J et al. Blockade of peripheral neuronal barrage reduces postoperative pain. Pain 1997; 70:209-215.

29. Banic B, Petersen-Felix S, Andersen OK et al. Evidence for spinal cord hypersensitivity in chronic pain after whiplash injury and in fibromyalgia. Pain 2004; 107:7-15.

30. Curatolo M, Petersen-Felix S, ArendtNielsen L et al. Central hypersensitivity in chronic pain after whiplash injury. Clin J Pain 2001; 17:306-315.

31. Paqueron $X$, Leguen $M$, Gentili ME et al. Influence of sensory and proprioceptive impairment on the development of phantom limb syndrome during regional anesthesia. Anesthesiology 2004; 100:979-986.

32. Paqueron X, Gentili ME, Willer JC et al. Time sequence of sensory changes after upper extremity block: swelling sensation is an early and accurate predictor of success. Anesthesiology 2004; 101:162-168.

33. Hogan QH, Abram SE. Neural blockade for diagnosis and prognosis. A review. Anesthesiology 1997; 86:216-241.

34. Benrath J, Brechtel C, Martin E et al. Low doses of fentanyl block central sensitization in the rat spinal cord in vivo. Anesthesiology 2004; 100:1545-1551.

35. Eichenberger U, Giani C, Petersen-Felix S et al. Lumbar epidural fentanyl: segmental spread and effect on temporal summation and muscle pain. Br / Anaesth 2003; 90: 467-473.

36. Tegeder I, Meier S, Burian M et al. Peripheral opioid analgesia in experimental human pain models. Brain 2003; 126:10921102.

37. Fanelli G, Casati A, Beccaria P et al. A double-blind comparison of ropivacaine, bupivacaine, and mepivacaine during sciatic and femoral nerve blockade. Anesth Analg 1998; 87:597-600.

38. Casati A, Fanelli G, Borghi B et al. Ropivacaine or $2 \%$ mepivacaine for lower limb peripheral nerve blocks. Study Group on Orthopedic Anesthesia of the Italian Society of Anesthesia, Analgesia, and Intensive Care. Anesthesiology 1999; 90:10471052.

39. Lund C, Selmer P, Hansen DB et al. Effects of epidural bupivacaine on somatosensory evoked potentials after dermatomal stimulation. Anesth Analg 1987; 66:34-38.

40. Malmqvist L, Tryggvason B, Bengtsson M. Sympathetic blockade during extradural analgesia with mepivacaine or bupivacaine. Acta Anaesthesiol Scand 1989; 33: 444-449.

41. Ford DJ, Raj PP, Singh P et al. Differential peripheral nerve block by local anesthetics in the cat. Anesthesiology 1984; 60:2833.

42. Fink BR, Cairns AM. Lack of size-related differential sensitivity to equilibrium conduction block among mammalian myelinated axons exposed to lidocaine. Anesth Analg 1987; 66:948-953.

43. Franz DN, Perry RS. Mechanisms of differential block among single myelinated and non-myelinated axons by procaine. J Physiol 1974; 236:193-210.

44. Lou L, Sabar R, Kaye A. Local Anesthetics. In Raj PP (ed). Textbook of Regional Anesthesia. Churchill Livingstone, Philadelphia 2002, pp 177-213.

45. Selander D, Sjostrand J. Longitudinal spread of intraneurally injected local anesthetics. An experimental study of the initial neural distribution following intraneural injections. Acta Anaesthesiol Scand 1978; 22:622-634.

46. Nakamura T, Popitz-Bergez F, Birknes J et al. The critical role of concentration for lidocaine block of peripheral nerve in vivo: studies of function and drug uptake in the rat. Anesthesiology 2003; 99:1189-1197.

47. Sung DH. Locating the target nerve and injectate spread in rabbit sciatic nerve block. Reg Anesth Pain Med 2004; 29:194200.

48. Popitz-Bergez FA, Leeson S, Strichartz GR, Thalhammer JG. Relation between functional deficit and intraneural local anesthetic during peripheral nerve block. A study in the rat sciatic nerve. Anesthesiology. 1995; 83:583-92.

49. Lambert LA, Lambert DH, Strichartz GR. Irreversible conduction block in isolated nerve by high concentrations of local anesthetics. Anesthesiology 1994; 80:10821093.

50. Devor M, Wall PD, Catalan N. Systemic lidocaine silences neuroma and DRG discharge without blocking nerve conduction. Pain 1992; 48:261-268.

51. Xavier AV, McDanal J, Kissin I. Relief of sciatic radicular pain by sciatic nerve block. Anesth Analg 1988; 67:1177-1180.

52. Klein SM, Eck J, Nielsen K, Steele SM.Anesthetizing the phantom: peripheral nerve stimulation of a nonexistent extremity. Anesthesiology 2004 Mar;100: 736-737. 
